\title{
Solução Numérica para PVI via aritmética fuzzy interativa: uma aplicação aos modelos epidemiológicos SI
}

\author{
Vinícius Francisco Wasques ${ }^{1}$ \\ Instituto de Matemática, Estatística e Computação Científica, UNICAMP, Campinas, SP \\ Estevão Esmi Laureano ${ }^{2}$ \\ Instituto de Matemática, Estatística e Computação Científica, UNICAMP, Campinas, SP \\ Laécio Carvalho de Barros ${ }^{3}$ \\ Instituto de Matemática, Estatística e Computação Científica, UNICAMP, Campinas, SP
}

\begin{abstract}
Resumo. Apresentamos neste trabalho soluções numéricas para PVI fuzzy, com aplicação em modelos epidemiológicos do tipo SI (Suscetível-Infectado) com dinâmica vital em que as condições iniciais são consideradas incertas e modeladas por números fuzzy. Nas equações diferenciais são consideradas derivadas para processos fuzzy autocorrelacionados. A fim de manipular as operações, são definidas aritméticas fuzzy interativa, baseada em uma família de distribuições de possibilidade conjunta parametrizada $J_{\gamma}$.
\end{abstract}

Palavras-chave. Interatividade Fuzzy, Distribuição de Possibilidade Conjunta, Problema de Valor Inicial Fuzzy.

\section{Introdução}

Os modelos matemáticos epidemiológicos são frequentemente estudados para compreender a dinâmica e a evolução de uma certa doença, e possibilita indicar algum controle para a mesma. Geralmente, os modelos matemáticos para doenças de transmissão direta são dados por sistemas de equações diferenciais ordinárias ou parciais. No entanto, os modelos clássicos não levam em conta incertezas provenientes de parâmetros ou de conhecimentos parciais, possivelmente presentes nos fenômenos biológicos.

Dentre os diversos modelos epidemiológicos conhecidos, abordaremos aquele do tipo SI (Suscetível $(S)$-Infectado $(I)$ ) com dinâmica vital, isto é, os indivíduos suscetíveis que contraem a doença não se recuperam. Um exemplo de doença que se comporta desse modo é a AIDS [5]. Tal modelo matemático é dado pelo sistema abaixo (1)

$$
\begin{cases}\frac{d S}{d t}=-\beta S I+(\eta-\mu) S, & S(0)=S_{0} \\ \frac{d I}{d t}=\beta S I-\mu I, & I(0)=I_{0}\end{cases}
$$

\footnotetext{
${ }^{1}$ viniwasques@hotmail.com

2eelaureano@gmail.com

${ }^{3}$ laeciocb@ime.unicamp.br
} 
com condições iniciais $S_{0}$ e $I_{0}$ fuzzy, sendo $\eta, \mu$ e $\beta$ as taxas de natalidade, mortalidade e de transmissão da doença, respectivamente.

Supondo que as condições iniciais $S_{0}$ e $I_{0}$ são incertas e modeladas por números fuzzy, existem algumas maneiras de se resolver um PVI fuzzy. Em [2] por exemplo, é utilizada uma correlação linear entre os números fuzzy em questão. No entanto, esse tipo de correlação exige que os números fuzzy em questão sejam de mesma forma: triangular, trapezoidal...Adotamos outro tipo de correlação, que pode ser aplicado para quaisquer números fuzzy. Aqui consideramos condições iniciais triangulares.

Para isto, utilizamos uma família de distribuições de possibilidade conjunta parametrizada, definida em [6], para levar em consideração a interatividade entre os números fuzzy envolvidos e determinar uma solução numérica para o sistema (1). Para tanto, o método de Euler é usado.

\section{Preliminares}

A seguir apresentamos alguns conceitos básicos da teoria de conjuntos fuzzy e algumas notações utilizadas.

Definição 2.1. [9] Um subconjunto fuzzy $A$ de $U$ é caracterizado por uma função de pertinência $\mu_{A}: U \rightarrow[0,1]$ em que $\mu_{A}(x)$ indica o grau com que $x$ pertence a $A$.

Todo subconjunto clássico A em U pode ser definido por sua função característica, isto é, pela função $\chi_{A}: U \rightarrow\{0,1\}$ que assume o valor 1 se $x \in A$ ou o valor 0 se $x \notin A$. Podemos dizer então que um conjunto clássico é em particular um conjunto fuzzy. Denotamos por $\mathcal{F}(U)$ a família de todos os subconjuntos fuzzy de $U$. Para facilitar a notação denotamos as funções de pertinência $\mu_{A}(x)$ por $A(x)$ para todo $x \in U$.

Os números fuzzy, denotados por $\mathbb{R}_{\mathcal{F}}$, são subconjuntos fuzzy dos reais que satisfazem às seguintes condições [1]:

(a) São normais, isto é, existe $x_{0} \in \mathbb{R}$ tal que $A\left(x_{0}\right)=1$;

(b) O suporte, $\operatorname{supp}(A)=\{x \in \mathbb{R}: A(x)>0\}$, é limitado;

(c) Os $\alpha$-níveis, $[A]^{\alpha}=\{x \in \mathbb{R}: A(x) \geq \alpha\}$, são intervalos fechados para todo $\alpha \in[0,1]$, e portanto serão denotados por $[A]^{\alpha}=\left[a_{\alpha}^{-}, a_{\alpha}^{+}\right]$.

Exemplos de números fuzzy são os números triangulares e trapezoidais, denotados por $(a ; b ; c)$ e $(a ; b ; c ; d)$, respectivamente [1].

Dentre o conjunto dos números fuzzy, destacamos o subconjunto $\mathbb{R}_{\mathcal{F}_{C}}$, que é formado por todos os números fuzzy que possuem as funções $a_{\alpha}^{-}$e $a_{\alpha}^{+}$contínuas [3].

Um conjunto fuzzy $J$, definido em um cartesiano $X=X_{1} \times X_{2} \times \ldots \times X_{n}$, é chamado de uma relação fuzzy n-ária. A projeção de uma relação fuzzy $J \in \mathcal{F}\left(X_{1} \times X_{2} \times \ldots \times X_{n}\right)$ sobre $X_{i}$, com $1 \leq i \leq n$, é o conjunto fuzzy $\prod_{J}^{i}$ de $X_{i}$ dado por

$$
\prod_{J}^{i}(y)=\bigvee\left\{J(x): x \in X \operatorname{com} x_{i}=y\right\}, \forall y \in X_{i}
$$


onde $\bigvee$ representa o sup.

Uma relação fuzzy $J \in \mathcal{F}\left(\mathbb{R}^{n}\right)$ é dita distribuição de possibilidade conjunta dos números fuzzy $A_{1}, \ldots, A_{n}$ se $A_{i}=\prod_{J}^{i}, \forall i=1, \ldots, n$. Ainda, os números fuzzy $A_{1}, \ldots, A_{n} \in \mathbb{R}_{\mathcal{F}}$ são ditos não interativos se, e somente se, a sua distribuição de possibilidade conjunta é dada por

$$
J\left(x_{1}, \ldots, x_{n}\right)=\bigwedge_{i=1}^{n} A_{i}\left(x_{i}\right),
$$

para todo $\left(x_{1}, \ldots, x_{n}\right) \in \mathbb{R}^{n}$. Caso contrário, dizemos que $A_{1}, \ldots, A_{n} \in \mathbb{R}_{\mathcal{F}}$ são interativos. O símbolo $\bigwedge$ representa o mínimo.

Um exemplo de interatividade entre números fuzzy é dado pelo conceito de completamente correlacionado [4]. Dizemos que $A_{1}, A_{2} \in \mathbb{R}_{\mathcal{F}}$ são completamente correlacionados em termos da distribuição de possibilidade conjunta $\mathrm{J}$ se existem $q, r \in \mathbb{R} \operatorname{com} q \neq 0$ tal que

$$
\begin{aligned}
J\left(x_{1}, x_{2}\right)=J_{q, r}\left(x_{1}, x_{2}\right) & =A_{1}\left(x_{1}\right) \chi_{\left\{q x_{1}+r=x_{2}\right\}}\left(x_{1}, x_{2}\right) \\
& =A_{2}\left(x_{2}\right) \chi_{\left\{q x_{1}+r=x_{2}\right\}}\left(x_{1}, x_{2}\right)
\end{aligned},
$$

para todo $x_{1}, x_{2} \in \mathbb{R}$.

Esta distribuição de possibilidade conjunta é restritiva, uma vez que exclui números fuzzy que não possam ser correlacionados através de uma reta, como é o caso entre um número fuzzy triangular e um trapezoidal.

Definição 2.2. (Princípio de Extensão Sup-J [7]) Sejam $J \in \mathcal{F}\left(\mathbb{R}^{n}\right)$ uma distribuição de possibilidade conjunta de $A_{1}, \ldots, A_{n} \in \mathbb{R}_{\mathcal{F}}$ e $f: \mathbb{R}^{n} \rightarrow \mathbb{R}$. A extensão sup-J da função $f$ aplicada em $\left(A_{1}, \ldots, A_{n}\right)$ é definida por

$$
f_{J}\left(A_{1}, \ldots, A_{n}\right)(y)=\bigvee_{\left(x_{1}, \ldots, x_{n}\right) \in f^{-1}(y)} J\left(x_{1}, \ldots, x_{n}\right),
$$

onde $f^{-1}(y)=\left\{\left(x_{1}, \ldots, x_{n}\right) \in \mathbb{R}^{n}: f\left(x_{1}, \ldots, x_{n}\right)=y\right\}$ é a pré-imagem de y pela função $f$.

Através da Definição 2.2 , é possível obter a soma interativa entre números fuzzy $A_{1} \mathrm{e}$ $A_{2}$ dada por

$$
\left(A_{1}+{ }_{J} A_{2}\right)(y)=\bigvee_{x_{1}+x_{2}=y} J\left(x_{1}, x_{2}\right),
$$

em que $f\left(x_{1}, x_{2}\right)=x_{1}+x_{2}$ e J uma distribuição de possibilidade conjunta de $A_{1}$ e $A_{2}$.

Definiremos a seguir uma distribuição de possibilidade conjunta conveniente, a fim de apresentar uma solução para o sistema (1) via método de Euler.

Considerando $\gamma \in[0,1]$, o parâmetro que estipula o grau de interatividade entre $A_{1}, A_{2} \in \mathbb{R}_{\mathcal{F}}$, construímos uma família de distribuições de possibilidade conjunta $J_{\gamma}$, da seguinte forma [8]. Sejam as funções auxiliares $g_{\wedge}^{i}, g_{\vee}^{i}$ e $v^{i}$, definidas por,

$$
g_{\wedge}^{i}(z, \alpha)=\bigwedge_{w \in\left[A_{3-i}\right]^{\alpha}}|w+z|, \forall z \in \mathbb{R}
$$




$$
g_{\vee}^{i}(z, \alpha)=\bigvee_{w \in\left[A_{3-i}\right]^{\alpha}}|w+z|, \quad \forall z \in \mathbb{R}
$$

e

$$
v^{i}(z, \alpha, \gamma)=(1-\gamma) g_{\wedge}^{i}(z, \alpha)+\gamma g_{\vee}^{i}(z, \alpha), \quad \forall z \in \mathbb{R},
$$

para cada $i \in\{1,2\}$ e $\alpha, \gamma \in[0,1]$.

Considere também,

$$
R_{\alpha}^{i}=\left\{\begin{array}{cl}
\left\{a_{i_{\alpha}}^{-}, a_{i_{\alpha}}^{+}\right\} & \text {se } \alpha \in[0,1) \\
{\left[A_{i}\right]^{1}} & \text { se } \alpha=1
\end{array}\right.
$$

$\mathrm{e}$

$$
L^{i}(z, \alpha, \gamma)=\left[A_{3-i}\right]^{\alpha} \cap\left[-v^{i}(z, \alpha, \gamma)-z, v^{i}(z, \alpha, \gamma)-z\right] .
$$

Para cada par $A_{1}, A_{2} \in \mathbb{R}_{\mathcal{F}}$, definimos $J_{\gamma}$ por

$$
J_{\gamma}\left(x_{1}, x_{2}\right)=\left\{\begin{array}{cl}
A_{1}\left(x_{1}\right) \wedge A_{2}\left(x_{2}\right), & \text { se }\left(x_{1}, x_{2}\right) \in P(\gamma) \\
0, & \text { caso contrário }
\end{array}\right.
$$

com

$$
P(\gamma)=\bigcup_{i=1}^{2} \bigcup_{\alpha \in[0,1]} P^{i}(\gamma, \alpha)
$$

e $P^{i}(\gamma, \alpha)$ sendo definido para cada $i \in\{1,2\}$ e $\gamma, \alpha \in[0,1]$, por

$\forall \alpha \in[0,1]$

$$
P^{i}(\gamma, \alpha)=\left\{\left(x_{1}, x_{2}\right): x_{i} \in R_{\alpha}^{i} \text { e } x_{3-i} \in L^{i}\left(x_{i}, \alpha, \gamma\right)\right\}
$$

A aritmética interativa baseada na distribuição de possibilidade conjunta dada por (7) não possui restrições, uma vez que pode ser construída para quaisquer pares de números fuzzy.

Com objetivo de simplificar os cálculos das funções auxiliares (4), (5), (6) e diminuir o esforço computacional, introduzimos o conceito de translação para números fuzzy.

Teorema 2.1. [8] Dados $A_{1}, A_{2} \in \mathbb{R}_{\mathcal{F}}$ e $c=\left(c_{1}, c_{2}\right) \in \mathbb{R}^{2}$. Sejam $\tilde{A}_{1}, \tilde{A}_{2} \in \mathbb{R}_{\mathcal{F}_{C}}$ tais que $\tilde{A}_{1}(x)=A_{1}\left(x+c_{1}\right)$ e $\tilde{A}_{2}(x)=A_{2}\left(x+c_{2}\right), \forall x \in \mathbb{R}$.

Seja $\gamma \in[0,1]$ e $\tilde{J}_{\gamma}$ uma distribuição de possibilidade conjunta entre $\tilde{A}_{1}, \tilde{A}_{2} \in \mathbb{R}_{\mathcal{F}_{C}}$. Se $J_{\gamma}^{c}$ é a relação fuzzy definida por

$$
J_{\gamma}^{c}\left(x_{1}, x_{2}\right)=\tilde{J}_{\gamma}\left(x_{1}-c_{1}, x_{2}-c_{2}\right), \quad \forall\left(x_{1}, x_{2}\right) \in \mathbb{R}^{2}
$$

então $J_{\gamma}^{c}$ é uma distribuição de possibilidade conjunta entre $A_{1}$ e $A_{2}$, ainda $\left(A_{1}+{ }_{\gamma}^{c} A_{2}\right) \in$ $\mathbb{R}_{\mathcal{F}_{C}}$, sendo $+_{\gamma}^{c}$ o símbolo que representa a soma via extensão sup $-J_{\gamma}^{c}$.

Pode-se mostrar que $\left[\tilde{A}_{1}+{ }_{\gamma} \tilde{A}_{2}\right]^{\alpha}-\left\{c_{1}+c_{2}\right\}=\left[A_{1}+{ }_{\gamma}^{c} A_{2}\right]^{\alpha}$ para todo $\alpha \in[0,1]$, sendo $+_{\gamma}$ o símbolo que representa a soma via extensão sup $-J_{\gamma}$. 


\section{Solução Numérica}

Como é bem conhecido, o método de Euler consiste em determinar soluções numéricas para equações diferenciais ordinárias como a descrita em (8)

$$
\left\{\begin{array}{l}
\frac{d x}{d t}=f(t, x) \\
x\left(t_{0}\right)=x_{0}
\end{array} .\right.
$$

O algoritmo de tal método é descrito por $x_{k+1}=x_{k}+h f\left(t_{k}, x_{k}\right)$, com $0 \leq k \leq N-1$, sendo $N$ o número de partições que o intervalo de tempo considerado é dividido e $h$ o tamanho dos subintervalos $\left[t_{k}, t_{k+1}\right]$ igualmente espaçados.

Utilizaremos este método a fim de obter uma solução numérica para o sistema (1). No entanto considerando que as condições iniciais são números fuzzy interativos, usaremos operações aritméticas no método de Euler que são provenientes de uma distribuição $J_{\gamma}$ adequada. Mais ainda, a noção de derivada (que considera operação de diferença no incremento) que aparece em (1) deve ser compatível com essa distribuição. Sendo assim, esse sistema é composto por derivadas interativas [2] e portanto a solução desse sistema é um processo fuzzy interativo.

As soluções numéricas para o PVI fuzzy (1) são dadas por (9)

$$
\left\{\begin{array}{l}
S_{k+1}=S_{k}+{ }_{\gamma}^{c} h\left(-\beta S_{k} *_{\gamma}^{c} I_{k}+{ }_{\gamma}^{c}(\eta-\mu) S_{k}\right) \\
I_{k+1}=I_{k}+{ }_{\gamma}^{c} h\left(\beta S_{k} *_{\gamma}^{c} I_{k}-{ }_{\gamma}^{c} \mu I_{k}\right)
\end{array},\right.
$$

sendo $+_{\gamma}^{c}, *_{\gamma}^{c} \mathrm{e}-{ }_{\gamma}^{c}$ a soma, produto e a diferença via extensão sup $-J_{\gamma}$, respectivamente.

Podemos ver nas Figuras 1, 2, 3 e 4 as soluções numéricas, cujos parâmetros utilizados foram $h=0.125, \beta=0.01, \eta=2.10^{-5}, \mu=1.10^{-5}$ e como condição inicial os números fuzzy triangulares $S_{0}=(1 ; 2 ; 3)$ e $I_{0}=(4 ; 5 ; 6)$.

Nos gráficos da dinâmica do modelo podemos comprovar que o parâmetro $\gamma$ está relacionado com o diâmetro da solução, isto é, para valores de $\gamma$ próximos de 0 o diâmetro diminui em relação ao tempo conforme as Figuras 1 e 2, em que $\gamma=0$ e $\gamma=0.25$ respectivamente. Por outro lado, para maiores valores de $\gamma$ o diâmetro aumenta, como é o caso das Figuras 3 e 4, nas quais $\gamma=0.5$ e $\gamma=0.75$, respectivamente. Isso ocorre pois, temos a seguinte equivalência [8] $\gamma_{1} \leq \gamma_{2} \Leftrightarrow J_{\gamma_{1}}^{c} \subseteq J_{\gamma_{2}}^{c} \Leftrightarrow A_{1}+_{\gamma_{1}}^{c} A_{2} \subseteq A_{1}+{ }_{\gamma_{2}}^{c} A_{2} \Leftrightarrow$ $\operatorname{diam}\left(A_{1}+{ }_{\gamma_{1}}^{c} A_{2}\right) \leq \operatorname{diam}\left(A_{1}+_{\gamma_{2}}^{c} A_{2}\right)$.
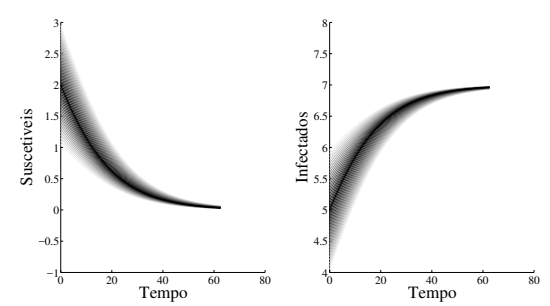

Figura 1: Solução numérica via extensão sup $-J_{0}$. As linhas em tons de cinza representam os $\alpha$-níveis. Os valores de $\alpha$ próximos de 1 são descritos pelas linhas mais escuras. 

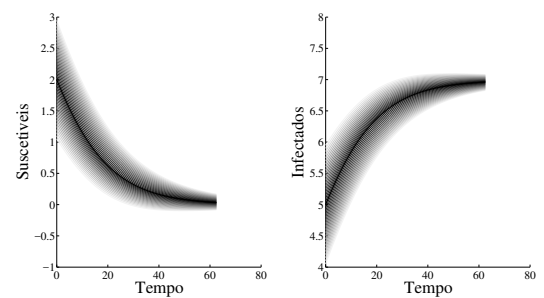

Figura 2: Solução numérica via extensão sup $-J_{0.25}$. As linhas em tons de cinza representam os $\alpha$-níveis. Os valores de $\alpha$ próximos de 1 são descritos pelas linhas mais escuras.
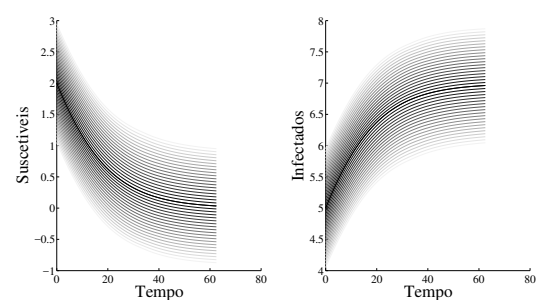

Figura 3: Solução numérica via extensão $s u p-J_{0.5}$. As linhas em tons de cinza representam os $\alpha$-níveis. Os valores de $\alpha$ próximos de 1 são descritos pelas linhas mais escuras.
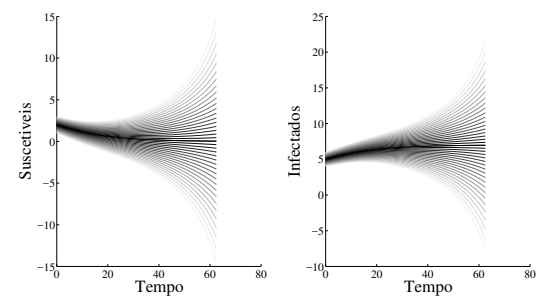

Figura 4: Solução numérica via extensão sup $-J_{0.75}$. As linhas em tons de cinza representam os $\alpha$-níveis. Os valores de $\alpha$ próximos de 1 são descritos pelas linhas mais escuras.

Do ponto de vista da doença, a interatividade entre as populações de suscetíveis e infectados é controlada pelo parâmetro $\gamma$, sendo que quanto mais próximo de 0 for este valor, maior é a interatividade.

O diâmetro de um número fuzzy está atrelado a incerteza que o mesmo modela, isto é, quanto menor for o valor do diâmetro, menor é a incerteza. Dito isso, percebemos que nas Figuras 1 e 2 o diâmetro da solução numérica diminui, e portanto nesses casos obtemos aproximações mais precisas da evolução temporal das populações em relação à doença. 


\section{Conclusão}

Consideramos um sistema epidemiológico SI, com dinâmica vital e condições iniciais incertas modeladas por números fuzzy interativos, obtendo um PVI fuzzy com derivadas interativas. Apresentamos soluções numéricas para este modelo, via método de Euler.

A partir de uma aritmética interativa, baseada no princípio de extensão sup $-J_{\gamma}$, controlamos a incerteza presente na dinâmica da doença através do parâmetro $\gamma$. As simulações computacionais corroboraram os resultados teóricos, pois é possível observar que o diâmetro da solução esta ligado a $\gamma$, de modo que para pequenos valores de $\gamma$ o diâmetro da solução numérica diminui, obtendo assim aproximações mais precisas da evolução temporal das populações em relação à doença.

Observamos também que através da distribuição $J_{\gamma}$ podemos efetuar operações aritméticas para quaisquer números fuzzy, o que não ocorre em geral para outras distribuições conhecidas na literatura, como por exemplo a distribuição para números fuzzy completamente correlacionados.

\section{Referências}

[1] L. C. Barros, e R. C. Bassanezi. Tópicos de Lógica Fuzzy e Biomatemática. IMECCUnicamp. Segunda Edição, 2010.

[2] L. C. Barros, and F. S. Pedro. Fuzzy differential equations with interactive derivative. Fuzzy Sets and Systems, 2016.

[3] B. Bede. Mathematics of Fuzzy Sets and Fuzzy Logic, ser. Studies in Fuzziness and Soft Computing. Springer, volume 295, 2013.

[4] C. Carlsson, R. Fullér, and P. Majlender. Additions of completely correlated fuzzy numbers. In Fuzzy Systems. Proceedings IEEE International Conference on, volume 1, pages 535-539, 2004.

[5] L. Edelstein-Keshet, Mathematical Models in Biology. Random House, New York, 1988.

[6] E. Esmi, G. Barroso, L. C. Barros, and P. Sussner. A new family of joint possibility distribution for adding interactive fuzzy numbers with applications to fuzzy initial value problems. 2015, submitted for publication.

[7] R. Fullér, and P. Majlender. On interactive fuzzy numbers. Fuzzy Sets and Systems, volume 143, number 3, pages 355-369, 2004.

[8] P. Sussner, E. Esmi, and L. C. Barros. Controling the Width of the Sum of Interactive Fuzzy Numbers with Applications to Fuzzy Initial Value Problems. Fuzzy Systems IEEE International Conference on, pages 1453-1460, 2016.

[9] L. A. Zadeh. Fuzzy Sets. Information and Control, volume 8, number 3, pages 338353, 1965. 